Check for updates

Cite this: Phys. Chem. Chem. Phys. 2020, 22, 11967

Received 23rd March 2020 Accepted 5th May 2020

DOI: 10.1039/d0cp01570a

rsc.li/pccp

\section{Solvent similarity index $\uparrow$}

\author{
Mark D. Driver (iD) and Christopher A. Hunter (D)*
}

The Solvent Similarity Index (SSI) is a quantitative parameter we introduce for the comparison of the solvation properties of any solvent or solvent mixture. The Surface Site Interaction Model for Liquids at Equilibrium (SSIMPLE) was used to calculate the free energy of solvation of a single Surface Site Interaction Point (SSIP) on a solute. The SSIP representation of molecular surfaces was used to calculate the free energy of solvation for all possible solute polarities, generating a unique solvation profile for any solvent or solvent mixture. Quantitative comparison of the solvation profiles of two solvents was used as the basis for calculating the solvation similarity index. Values of SSI were calculated for all pairwise comparisons of 261 pure solvents at $298 \mathrm{~K}$, and the results were used to classify solvents into groups according to their solvation properties. Applications to understanding the solvation properties of binary solvent mixtures and for identification of alternative solvents are illustrated.

\section{Introduction}

Solvent selection is a key issue for a very wide range of chemical processes. ${ }^{1,2}$ The reaction medium plays a key role in synthesis of pharmaceutics and agrochemicals, and solvent properties are fundamental to formulation of surface coatings, paints, and personal care products. $^{3-10}$ There are many considerations in choosing the best solvent, including cost and environmental effects, but the fundamental requirement is that the solvent makes the required interaction with solutes, leading to dissolution, precipitation, stabilisation or destabilisation. A combination of environmental and availability issues have encouraged the search for new alternative solvents. ${ }^{1,2,11-14}$ A range of scales have been devised to quantify and classify solvent properties. Many are based on experimental measurement of the strength of interactions with solutes or more indirectly the effect on the spectroscopic properties of solutes, ${ }^{4,15}$ and others are based on more qualitative descriptors such as the environmental impact of the solvent. ${ }^{1,2,11-14}$ The commonly used Hansen solubility parameter is based on miscibility, which is based on the idea that like dissolves like and so miscible solvents should also dissolve the same solutes. ${ }^{4}$

Calculation of solvation free energies has been extensively explored with multiple different approaches: ${ }^{16-18}$ empirical functions, implicit solvation simulations or explicit solvation simulations. Empirical methods consist of parameterised

Department of Chemistry, University of Cambridge, Lensfield Road, Cambridge CB2 1EW, UK. E-mail: herchelsmith.orgchem@ch.cam.ac.uk; Tel: +44 (0)1223 336710

$\dagger$ Electronic supplementary information (ESI) available: PDF file containing SSIP normalisation domains, a list of solvents used in this study, and details of the solvent clusters from the dendrogram in Fig. 2, and a csv file of pure solvent SSI values. See DOI: 10.1039/d0cp01570a functions that use the correlation of molecular properties to experimental measurement. These functions either use the summation of information for individual fragments in the chemical structure in group contribution type methods, ${ }^{19-31}$ or quantitative structure property relationships (QSPR) where molecular descriptors are used. ${ }^{32-37}$ For implicit solvation methods the solvent medium is treated as a dielectric continuum. ${ }^{38-40}$ Parameterised relationships are then used to convert the activity coefficients generated from such calculations to free energies. ${ }^{41-46}$ Full atomic simulations are required for explicit solvation models, using molecular dynamics (MD) or Monte Carlo (MC) frameworks to propagate a system in phase space. Calculation of free energies requires summation of the free energy components of interactions between solute and solvent. ${ }^{47-54}$

Here we describe a new solvent similarity index, which quantifies the similarity between two solvents based on calculation of solvation free energies using the Surface Site Interaction Model for Liquids at Equilibrium (SSIMPLE). ${ }^{55}$

Solvent-solute interactions play an important role in dissolution and solvation of compounds. Solvents are used in several industries, as a medium for reactions to synthesise compounds such as in the pharmaceutical and agrochemical industries, or for the application of surface coatings and paints. Finding the solvent with the correct properties to solvate the required components is therefore important. Multiple solvents may possess similar efficacy at solvation, so secondary factors such as cost and environmental effects also play a role in selection. With the increasing desire to reduce environmental impact and improve efficiency in processes, the concept of 'green' chemistry $^{56}$ is becoming more prominent, with scales to assess the environmental impact of solvents developed. ${ }^{11-14}$ This has led to the use of alternative solvents with lower environmental impact in pharmaceutical companies. ${ }^{11,57}$ 


\section{The SSIMPLE approach}

The approach is based on description of molecules as a set of Surface Site Interaction Points (SSIPs), which can be used to describe all of the non-covalent interactions that the molecule makes with the environment, e.g. the solvent. Each SSIP is assigned an interaction parameter (referred to as an SSIP value in this work), $\varepsilon_{i}$, which is equivalent to the experimentally measured hydrogen bond donor parameter $(\alpha)$ for positive sites or the hydrogen bond acceptor parameter $(-\beta)$ for negative sites. ${ }^{58}$ These experimental hydrogen bond parameters were originally derived from the $\alpha_{2}^{\mathrm{H}}$ and $\beta_{2}^{\mathrm{H}}$ scales developed by Taft and Abraham, ${ }^{9,59-61}$ who used experimental measurements of association constants for formation of a wide variety of 1:1 hydrogen bonded complexes in nonpolar solvents. Murray and Politzer ${ }^{62-64}$ have shown that non-covalent interactions can be rationalised based on the properties of calculated molecular electrostatic potential surfaces (MEPS). We have shown that the experimentally determined hydrogen bond parameters correlate rather well with the calculated MEPS, and this correlation forms the basis for the SSIP approach described here. The assignment of the calculated SSIP values is done by footprinting of the ab initio calculated MEPS of the isolated molecule in the gas phase as described previously. ${ }^{65}$

To describe a liquid, SSIP interactions are treated in a pairwise manner, such that the association constant for interaction between the $i$ th and $j$ th SSIP, $K_{i j}$, is given by eqn (1).

$$
K_{i j}=\frac{1}{2} \mathrm{e}^{-\frac{\varepsilon_{i} \varepsilon_{j}+E_{\mathrm{vdW}}}{R T}}
$$

where $E_{\mathrm{vdw}}=-5.6 \mathrm{~kJ} \mathrm{~mol}^{-1} \cdot 66$

The interaction energy is made up of a polar term, $\varepsilon_{i} \varepsilon_{j}$, and a non-polar term, $E_{\mathrm{vdw}}$, which is the energy of the van der Waals interaction between two SSIPs. For repulsive interactions (i.e. $\varepsilon_{i}$ and $\varepsilon_{j}$ have the same sign), it is assumed that a state can be found where the polar sites are misaligned such that only nondirectional van der Waals interactions are made, and the polar interaction term, $\varepsilon_{i} \varepsilon_{j}$, is set to zero. As we have shown previously, ${ }^{55,66}$ van der Waals interactions between non-polar molecules are a linear function of surface area, so by choosing a description that gives all SSIPs the same area footprint on the van der Waals surface of a molecule, a constant value can be used for $E_{\mathrm{VdW}}$. The standard state used to ensure $K_{i j}$ is dimensionless is the maximum theoretical density of SSIPs, $c_{\max }=$ $300 \mathrm{M}$. The value of $c_{\text {max }}$ is based on the volume an SSIP, $5 \AA^{3}$, that was defined using the volume enclosed by the van der Waals surface of a water molecule, which is represented by 4 SSIPs. ${ }^{55}$ The speciation of all SSIP contacts in the liquid phase can then be calculated.

The free energy of solvation of an SSIP that represents a solute, $\Delta G_{\mathrm{S}}$, can be calculated by considering the concentration of this SSIP that is not bonded to a solvent SSIP ([1 $\left.\left.1_{\mathrm{nb}}\right]\right) . \Delta G_{\mathrm{S}}$ in eqn (2) is the free energy of transfer of the solute SSIP from a reference state, which corresponds to a dilute gas where there are no SSIP interactions.

$$
\Delta G_{\mathrm{S}}=R T \ln \left(\frac{\left[1_{\mathrm{nb}}\right]}{[1]}\right)-R T \ln \left(\frac{\sqrt{1+8 \theta}-1}{4 \theta}\right)
$$

where [1] is the total concentration of the solute SSIP in the phase, and $\theta$ is the total SSIP concentration in the phase.

The first term in eqn (2) describes the interactions made by the solute SSIP with the solvent SSIPs. The second term in eqn (2) corrects for the increased probability of interaction between SSIPs when they are confined to a condensed phase..$^{55}$ The confinement energy is derived by considering a phase with an SSIP concentration of $\theta$, in which all pairwise SSIP contacts have an equilibrium constant $K_{i j}$ of one.

\section{Results}

The SSIP representation of 261 solvent molecules was described previously (see ESI $\dagger$ for full list of solvents). ${ }^{67}$ These representations were used to calculate solvation free energies for all solute SSIP values between -10 and +5 in increments of 0.1 . Plotting the results gives a unique solvation profile for each solvent, which describes the non-covalent interactions the solvent would make with any solute taking into account the polarity and the concentration of the interaction sites present in the solvent. Note that the effects of charge-charge interactions between ionic solutes is not described by SSIMPLE, so dielectric constant would be an important additional parameter for describing the solvation properties of ionic or ionisable solutes. The calculation of SSIP values was parameterised using experimentally determined free energy changes for formation of $1: 1$ hydrogen bonded complexes at $298 \mathrm{~K}$, so all of the calculations described here were also carried out at $298 \mathrm{~K}$.

\subsection{Solvation profiles}

Fig. 1 shows plots of solvation free energies $\left(\Delta G_{\mathrm{S}}\right)$ as a function of solute SSIP value $(\varepsilon)$ for three solvents. Toluene is a non-polar solvent, so has poor interactions with hydrogen bond donors and acceptors, leading to low solvation energies for all solutes, and the solvation profile is a relatively shallow curve. Dimethyl sulfoxide (DMSO) is a polar aprotic solvent. It has a strong hydrogen bond acceptor, so it solvates donors very strongly, with large negative values of $\Delta G_{\mathrm{S}}$ for positive SSIPs. DMSO is a weak donor, so it solvates acceptors poorly with small negative values of $\Delta G_{\mathrm{S}}$ for negative SSIPs. Water is a polar protic solvent with both hydrogen bond donors and acceptors. Thus water solvates hydrogen bond donor and hydrogen bond acceptor solutes reasonably well, leading to large negative values of $\Delta G_{\mathrm{S}}$ for strong hydrogen bond donors and acceptors. However, the value of $\Delta G_{\mathrm{S}}$ is positive for water when the value of the solute SSIP is zero $\left(+0.8 \mathrm{~kJ} \mathrm{~mol}^{-1}\right)$. The reason is that solvation of non-polar solutes requires breaking of water-water hydrogen bonds, and the loss of these polar interactions is not compensated by new polar interactions made with the solute. The solvation of non-polar solutes is therefore unfavourable for water, but favourable in the other two solvents, which have non-polar interaction sites available 


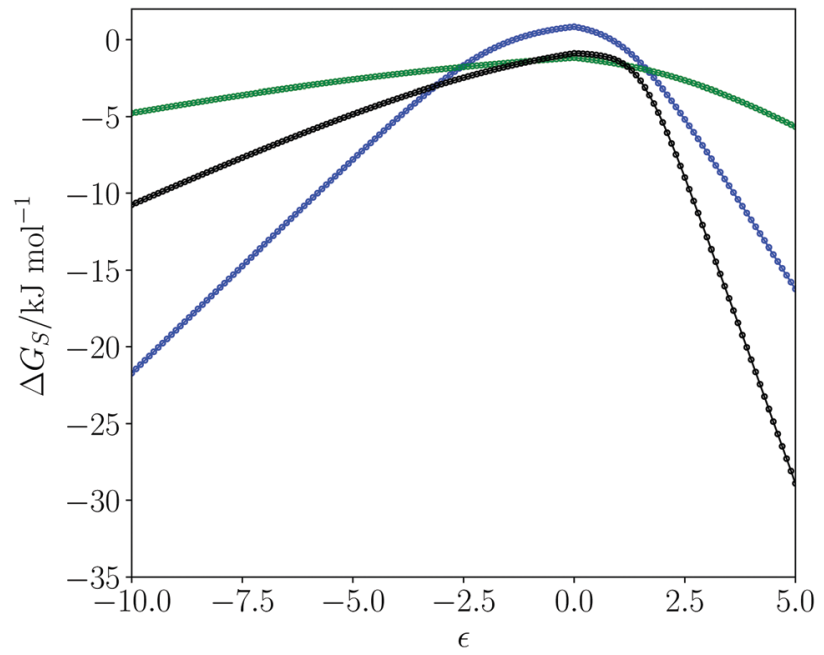

Fig. 1 Free energy of solvation $\left(\Delta G_{S}\right.$ in $\left.\mathrm{kJ} \mathrm{mol}^{-1}\right)$ of a single solute SSIP as a function of solute SSIP value $(\varepsilon)$ for water (blue), DMSO (black) and toluene (green) at $298 \mathrm{~K}$. The curves correspond to the solvation profiles of the solvents.

for interaction with a non-polar solute. We have previously shown that the solvation energies calculated using the SSIMPLE model provide an accurate quantitative description of the hydrophobic effect as measured by phase transfer free energies. ${ }^{55}$

\subsection{The solvent similarity index}

Comparison of the solvation profiles of different solvents requires definition of a metric which describes the distance between two curves for all possible solute SSIP values. The root mean square deviation (RMSD) between the solvation profiles for two solvents S1 and S2 is given by eqn (3).

$$
\operatorname{RMSD}(\mathrm{S} 1, \mathrm{~S} 2)=\sqrt{\frac{\sum_{N}\left(\Delta G_{\mathrm{S} 1}-\Delta G_{\mathrm{S} 2}\right)^{2}}{N}}
$$

where $N$ is the number of solute SSIP values used.

If the summation were carried out over the full curve then the RMSD would be dominated by the large solvation energy differences for the extreme ends of the $\varepsilon$ scale and would not capture the smaller differences in the non-polar region, which represents the most common hydrocarbon features of organic molecules. To avoid this issue, the curves were partitioned, and each domain was given an equal weighting after normalisation. The six domains given by eqn (4) were chosen to represent strong, moderate and weak donors and acceptors.

$$
\text { Domains }=\left\{\begin{array}{l}
-10.0 \leq \varepsilon<-5.0 \\
-5.0 \leq \varepsilon<-2.0 \\
-2.0 \leq \varepsilon<0.0 \\
0.0 \leq \varepsilon<1.0 \\
1.0 \leq \varepsilon<3.0 \\
3.0 \leq \varepsilon<5.0
\end{array}\right.
$$

The values of RMSD for each domain were scaled so that they carry equal weight. The normalisation factor for the $j$ th domain, $\eta_{j}$, is defined as the maximum RMSD between any two solvents in the set of all solvents for the domain. This results in scaled values between 0 and 1 for each domain, where 0 means the domains are identical, and a value of 1 means the domains are the most dissimilar of all pairings of solvents. We define the Solvent Similarity Index (SSI) as the mean of the scaled values of all domains (eqn (5)).

$$
\operatorname{SSI}(k, l)=\frac{1}{6} \sum_{j=1}^{6} \frac{\operatorname{RMSD}(k, l)}{\eta_{j}}
$$

where $k$ and $l$ refer to two different solvents.

The values of SSI depend on the values of $\eta_{j}$, which depend on which set of solvents is used to calculate them. Thus the definition of SSI provides a qualitative guide to similarity within a collection of solvent profiles. A quantitative measure can be created by defining normalisation factors, $\eta_{j}$ based on a standard reference set of solvents at a specified temperature. The set of 261 pure solvent molecules listed in the ESI $\uparrow$ were chosen as this reference set, with solvation calculations undertaken at $298 \mathrm{~K}$, and the solvents defining the extreme ranges are shown in Table 1. Hydrogen fluoride has the strongest hydrogen bond donor SSIP in the set of solvents and appears as an extreme solvent for solvation of strong and moderate hydrogen bond acceptors. Hexamethyl phosphoramide (HMPA) has the strongest hydrogen bond acceptor SSIP in the set of solvents and appears as an extreme solvent for solvation of strong and moderate hydrogen bond donors. Water is an extreme solvent for the solvation of non-polar domains, due to the hydrophobic effect. The solvents at the other ends of these extremes in Table 1 are a more heterogeneous collection that represent a complex function of the SSIP composition. Glycerol appears twice, which suggests that this solvent has some rather unique properties.

\subsection{Solvent similarities}

SSI values were calculated for all pairwise combinations of all 261 solvents in the reference set. A dendrogram showing the results is presented in Fig. 2. This diagram provides an overview of the similarities for all solvents in the set. Branch nodes in the dendrogram can be used to select threshold SSI values for classifying solvents into clusters with similar properties. The numbered branch nodes highlighted in Fig. 2 were used to define 14 distinct solvent regions (see ESI $\dagger$ for full details).

Many of the solvent similarity clusters in the dendrogram in Fig. 2 coincide with standard functional group classifications.

Table 1 Most dissimilar solvents used to calculate the normalisation factors, $\eta_{j}$, for each domain

\begin{tabular}{ll}
\hline Domain & Extreme solvents \\
\hline$-10.0 \leq \varepsilon<-5.0$ & Hydrogen fluoride and $n$-dodecane \\
$-5.0 \leq \varepsilon<-2.0$ & Hydrogen fluoride and glycerol \\
$-2.0 \leq \varepsilon<0.0$ & Thionyl chloride and water \\
$0.0 \leq \varepsilon<1.0$ & Glycerol and water \\
$1.0 \leq \varepsilon<3.0$ & HMPA and perfluoromethylcyclohexane \\
$3.0 \leq \varepsilon<5.0$ & HMPA and perfluoromethylcyclohexane
\end{tabular}




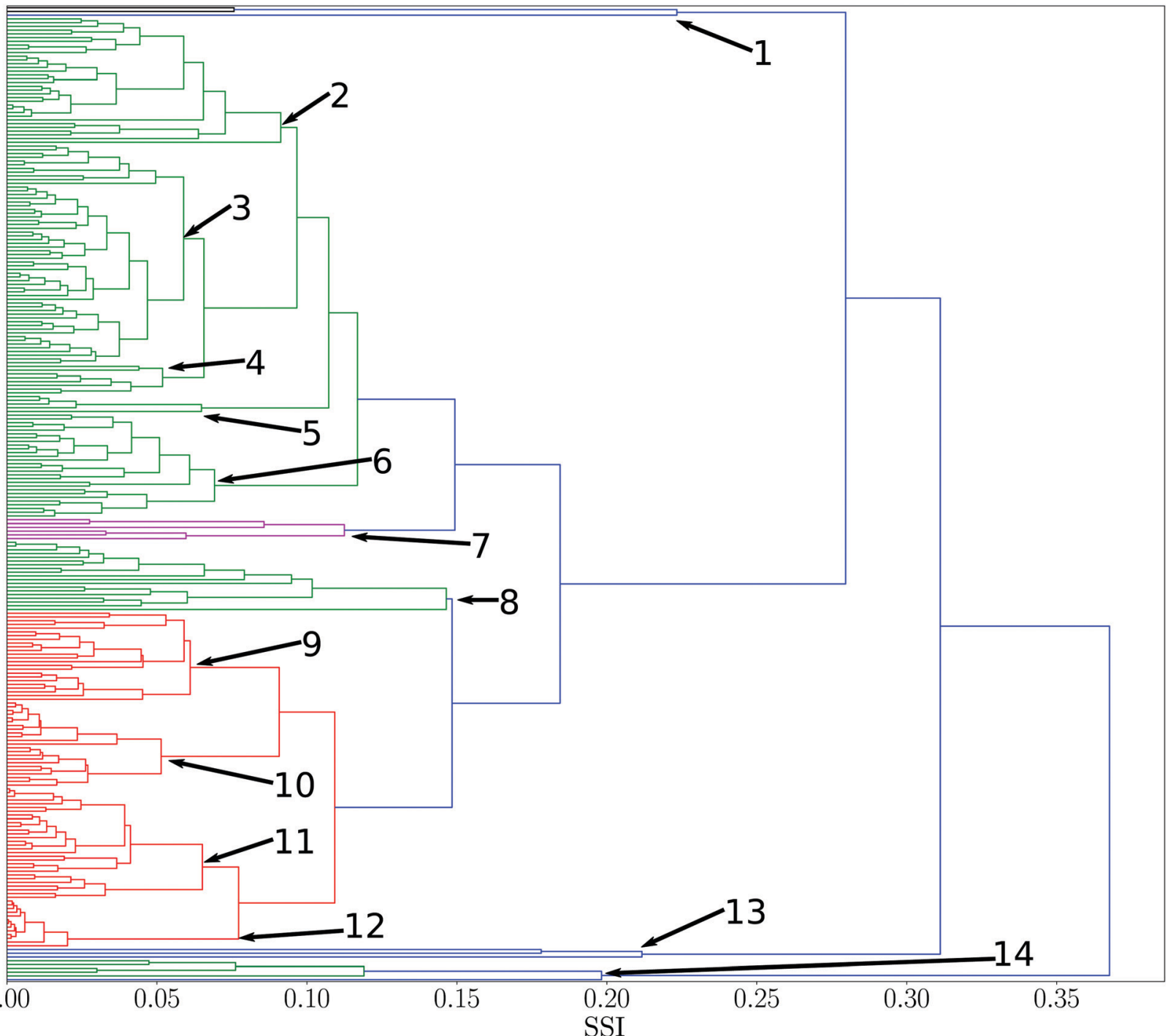

Fig. 2 Dendrogram showing solvent similarity indices (SSI) for 261 pure solvents at $298 \mathrm{~K}$ using UPGMA clustering algorithm to group solvents. Numbers correspond to nodes used for partitions into smaller subsets. Solvent clusters with a normalised distance between nodes of less than two fifths of the maximum distance was used as a threshold to colour different clusters (see ESI† for full details of the solvents in each of the 14 nodes).

For example, Fig. 3 shows region 12. The alkane solvents are all clustered together in one branch of this region. The second branch in region 12 contains the other two very non-polar solvents in the set, tetramethylsilane and carbon disulfide. The remaining non-polar solvents are found in regions 10 and 11 . Region 10 has two distinct branches: the aromatic hydrocarbons are clustered in one group, and aryl ethers and sulfides make up the other. Region 11 contains the haloalkanes.

All of the solvents in region 2 are alcohols with the exception of $\mathrm{N}$-methyl formamide, which has a good hydrogen bond donor and a good acceptor SSIP with similar values to the most polar alcohol SSIPs. The ketones, esters and ethers are all clustered together in region 3 , because they have very similar hydrogen bond acceptor properties and the only hydrogen bond donors are the alkyl $\mathrm{CH}$ groups. Region 5 has two branches: carboxylic acids are clustered in one group, and the other branch is the only pyrrole in the set of solvents.
Some regions in the dendrogram in Fig. 2 contain mixtures of solvents with a more diverse set of functional groups. For example, Fig. 4 shows the solvents in region 13 . The feature that water, hydrogen peroxide and formamide have in common is that they all have high concentrations of good hydrogen bond donors and good hydrogen bond acceptors, which means that solvophobic effects are uniquely important for solvation of nonpolar solutes in these solvents. Region 14 contains the solvents which have the strongest hydrogen bond donors. The strongest hydrogen bond acceptor, hexamethylphosphoric triamide (HMPA), is found in region 1. Region 1 has two branches, but HMPA is unique in that it has the highest value of SSI at which the dendrogram branches to a single solvent.

\subsection{Distribution of SSI}

The distribution of SSI values for all pairwise comparisons of solvents is shown in Fig. 5. The maximum theoretical SSI for 


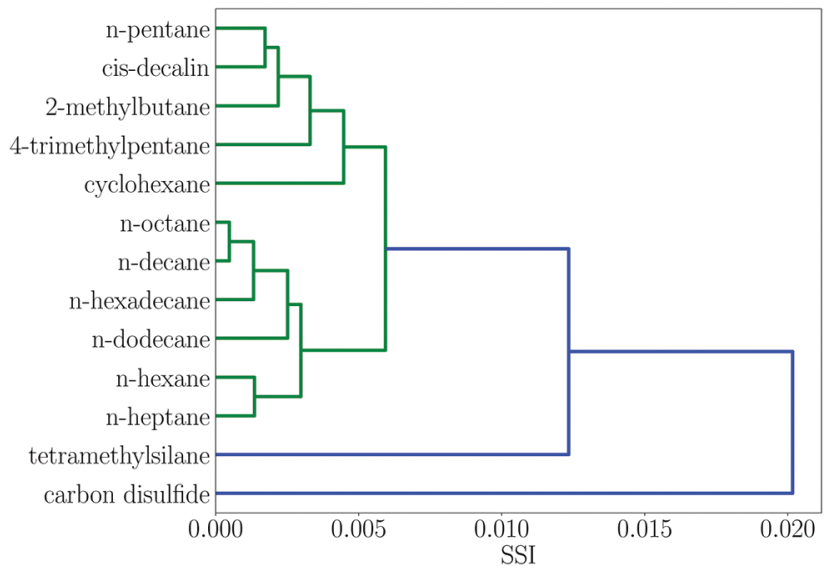

Fig. 3 Dendrogram showing solvent similarity indices (SSI) at $298 \mathrm{~K}$ for solvents to the left of node 12 in Fig. 2

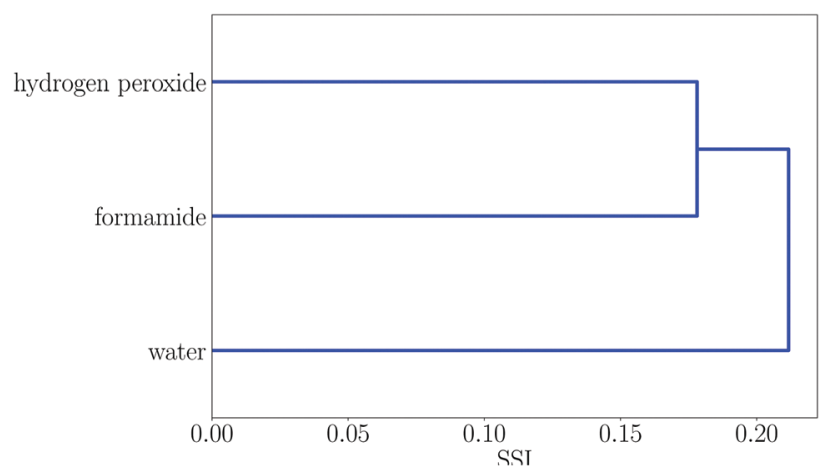

Fig. 4 Dendrogram showing solvent similarity indices (SSI) at $298 \mathrm{~K}$ for solvents to the left of node 13 in Fig. 2.

two solvents is 1.0, which would be obtained if a pair of solvents have the greatest dissimilarity in all six domains. The maximum value of SSI for the set of 261 solvents is 0.79 , for hydrogen fluoride and HMPA. Hydrogen fluoride contains a very good hydrogen bond donor, but poor hydrogen bond acceptors, so solvates hydrogen bond acceptors well and hydrogen bond donors poorly, whereas HMPA has the best hydrogen bond acceptor of any solvent but poor hydrogen bond donors, so exhibits the opposite solvation behaviour to hydrogen fluoride.

\subsection{Solvent mixtures}

By combining different solvents together as a mixture, a new solvent system with different properties is created. These mixtures possess properties that are a composite of the constituent parts. Comparison of the similarity of the solvent mixtures to the pure solvents provides a method to quantitatively measure the change in behaviour on mixing the solvents. Comparison of solvent mixtures with pure solvents can be used to develop a method based on SSI values for finding mixtures that might be used as substitutes for a specific pure solvent.

Table 2 shows the closest solvent for different water-ethanol compositions. As might be expected from the dendrogram in Fig. 4, hydrogen peroxide and formamide are the closest

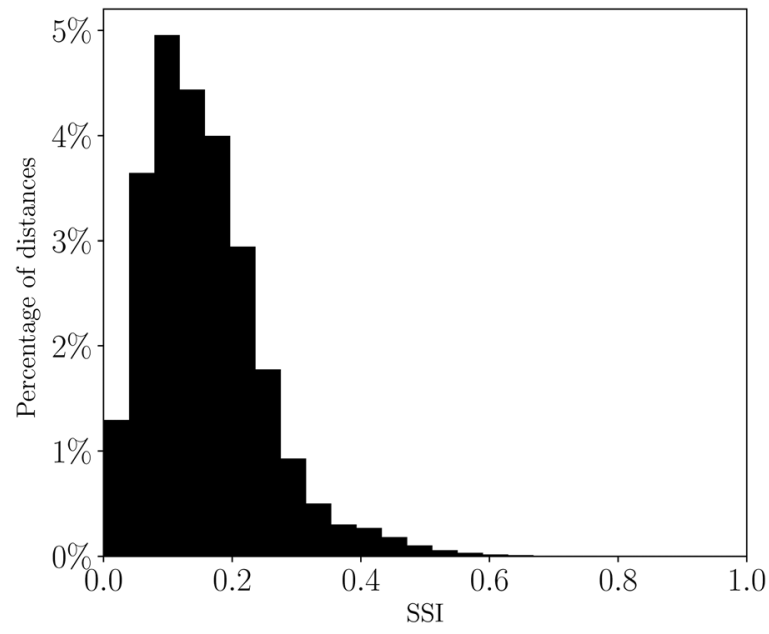

Fig. 5 Distribution of solvent similarity indices (SSI) at $298 \mathrm{~K}$ for all pairwise comparisons of 261 solvents plotted as a percentage of the total number of solvent pairs.

Table 2 Most similar solvents to water-ethanol mixtures ranked in order of decreasing solvent similarity index (SSI) calculated at $298 \mathrm{~K}$

\begin{tabular}{lll}
\hline Ethanol volume\% & Closest solvent & SSI \\
\hline 0 & Hydrogen peroxide & 0.194 \\
5 & Hydrogen peroxide & 0.171 \\
10 & Hydrogen peroxide & 0.152 \\
15 & Hydrogen peroxide & 0.137 \\
20 & Hydrogen peroxide & 0.126 \\
25 & Hydrogen peroxide & 0.119 \\
30 & Formamide & 0.111 \\
35 & Formamide & 0.096 \\
40 & Formamide & 0.083 \\
45 & Formamide & 0.071 \\
50 & Formamide & 0.060 \\
55 & Formamide & 0.052 \\
60 & Formamide & 0.047 \\
65 & Formamide & 0.048 \\
70 & Formamide & 0.055 \\
75 & Formamide & 0.065 \\
80 & Aminoethanol & 0.053 \\
85 & Aminoethanol & 0.039 \\
90 & Aminoethanol & 0.033 \\
95 & Allyl alcohol & 0.017 \\
100 & 1-Propanol & 0.015 \\
& &
\end{tabular}

solvents to the water-rich mixtures, due to the solvophobic properties. However, the minimum value of SSI for pure water is relatively high compared with the distribution in Fig. 5 ( 0.2 for hydrogen peroxide), highlighting the unique properties of aqueous solutions. For ethanol-rich mixtures, other short chain alcohols most closely resemble the mixed solvent, because there are similar concentrations of hydroxyl and alkyl SSIPs. Fig. 6 shows how the contributions from the 6 domains, which make up the value of SSI, vary with composition. The difference between the solvents is dominated by solvation of the non-polar SSIPs and varies uniformly with composition. Ethanol solvates non-polar functional groups well, due to preferential interactions with the solvent ethyl group. Water has no non-polar SSIPs, so the value of $\Delta G_{\mathrm{S}}$ for non-polar groups is positive for water. 


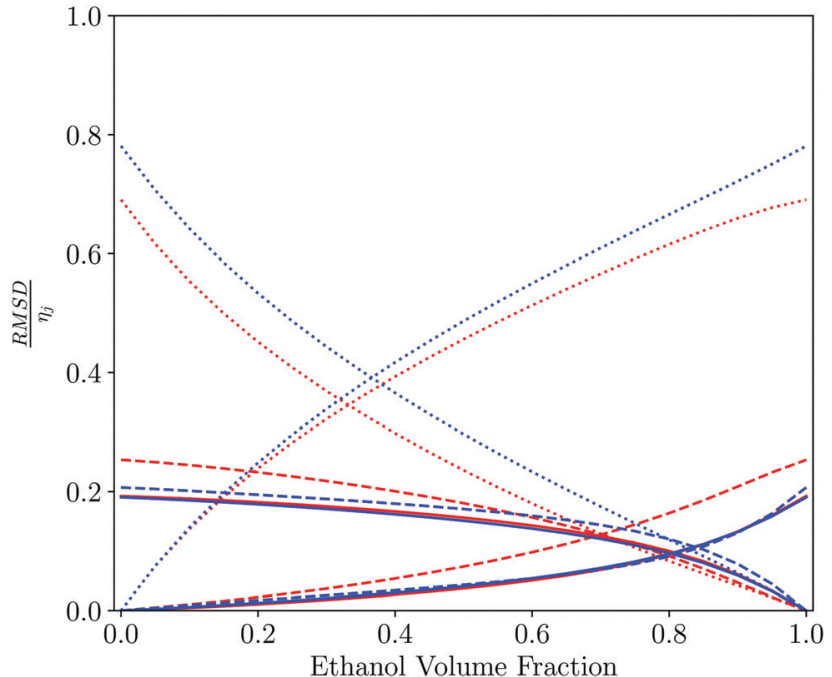

Fig. 6 Pairwise comparison of water-ethanol solvent mixtures with each of the pure components plotted as a function of the solvent composition of the mixture. Contribution to the total solvent similarity index (SSI) by domain, plotted as the normalised RMSD between the solvation profile of the mixture and the pure solvent, RMSD $/ \eta_{i j}$ as defined in eqn (3) and (4): strong acceptors (red solid line), moderate acceptors (red dashed line), weak acceptors (red dotted line), weak donors (blue dotted line), moderate donors (blue dashed line) and strong donors (blue solid line).

Table 3 and Fig. 7 show a similar analysis for mixtures of tetrahydrofuran (THF) and chloroform. In this case, a more diverse range of pure solvents give the closest match to the solvation properties of the mixtures. THF has a moderate hydrogen bond acceptor and no strong hydrogen bond donors, whereas chloroform has a moderate hydrogen bond donor and no strong hydrogen bond acceptors. Thus the THF-rich mixtures are most similar to solvents with a moderate hydrogen bond acceptor and no hydrogen bond donors, whereas the

Table 3 Most similar solvents to THF-chloroform mixtures ranked in order of decreasing solvent similarity index (SSI) calculated at $298 \mathrm{~K}$

\begin{tabular}{lll}
\hline Chloroform volume $\%$ & Closest solvent & SSI \\
\hline 0 & Morpholine & 0.028 \\
5 & Morpholine & 0.021 \\
10 & Morpholine & 0.016 \\
15 & Morpholine & 0.018 \\
20 & Morpholine & 0.024 \\
25 & Cyclohexanone & 0.023 \\
30 & Cyclohexanone & 0.017 \\
35 & Cyclohexanone & 0.022 \\
40 & Quinoline & 0.024 \\
45 & 3,3-Dimethyl-2-butanone & 0.024 \\
50 & Benzyl methyl ketone & 0.026 \\
55 & 2-Bromopyridine & 0.024 \\
60 & 2-Bromopyridine & 0.022 \\
65 & 3-Bromopyridine & 0.029 \\
70 & 3-Bromopyridine & 0.035 \\
75 & Benzonitrile & 0.037 \\
80 & Phenylacetonitrile & 0.039 \\
85 & Phenylacetonitrile & 0.045 \\
90 & Aniline & 0.047 \\
95 & 1,1,2-Trichloroethane & 0.046 \\
100 & Pentachloroethane & 0.017
\end{tabular}

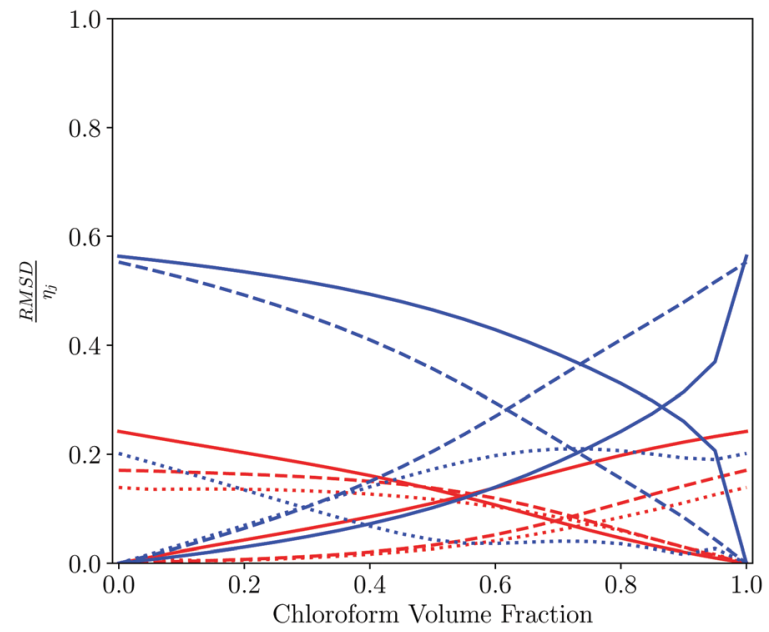

Fig. 7 Pairwise comparison of THF-chloroform solvent mixtures with each of the pure components plotted as a function of the solvent composition of the mixture. Contribution to the total solvent similarity index (SSI) by domain, plotted as the normalised RMSD between the solvation profile of the mixture and the pure solvent, RMSD/ $\eta_{i j}$ as defined in eqn (3) and (4): strong acceptors (red solid line), moderate acceptors (red dashed line), weak acceptors (red dotted line), weak donors (blue dotted line), moderate donors (blue dashed line) and strong donors (blue solid line).

chloroform-rich mixtures are most similar to solvents that also have polar $\mathrm{CH}$ groups. The contributions from each of the 6 domains used to calculate the SSI values for THF-chloroform mixtures are shown in Fig. 7. The major difference between the solvents is due to the way in which they solvate hydrogen bond donors.

\subsection{New solvents}

The SSI analysis provides a method for predicting the properties and potential utility of new solvents. Dihydrolevoglucosenone ((1R)-7,8-dioxabicyclo[3.2.1] octan-2-one, commercially known as cyrene) has been suggested as a green replacement for dimethyl formamide (DMF) and $N$-methyl pyrrolidine (NMP) ${ }^{68}$ Cyrene is produced from cellulose ${ }^{69}$ so the feedstock required can be supplied from a sustainable source of plant biomass. At the end of the lifecycle, the solvent can be incinerated without the release of $\mathrm{NO}_{x}$ or $\mathrm{SO}_{x}$. Cyrene has been shown to be a useable solvent for Sonogashira cross-coupling, Cacchi-type annulation ${ }^{70}$ and urea synthesis ${ }^{71}$ but has some sensitivity to basic conditions. ${ }^{70}$

Cyrene was included in the dendrogram of pure solvents and appears in region 4 (Fig. 2). DMF and NMP appear in region 6, and Fig. 8 shows a comparison of the solvation profiles. It is clear that solvation of hydrogen bond donors is not as good in cyrene compared with NMP or DMF. Table 4 shows the closest solvents to cyrene in rank order of SSI value. The SSI values in Table 4 are all significantly lower than the SSI to DMF (0.089) and to NMP (0.138). Cyrene, most closely matches 2,4-pentanedione. Fig. 8 illustrates the match between the solvation profiles for cyrene and acetone, which is one of the more common solvents in Table 4. Obviously, there are factors in addition to solvation properties that will come into play in solvent selection for a specific application: for example, the boiling points of cyrene and acetone are quite different. 


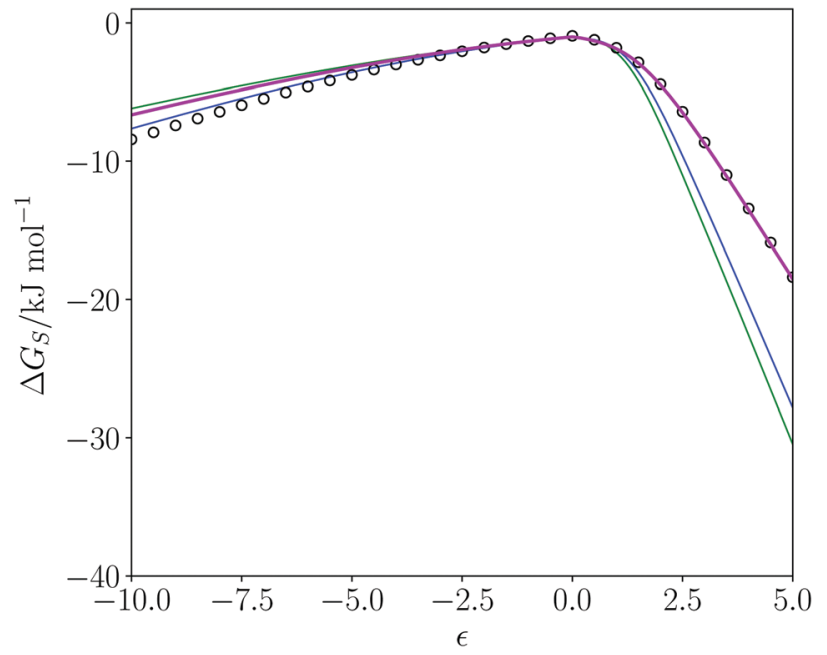

Fig. 8 Solvation profiles $\left(\Delta G_{S}\right.$ in $\mathrm{kJ} \mathrm{mol}^{-1}$ plotted as a function of solute SSIP value, $\varepsilon$ ) for dimethyl formamide (DMF) in blue, $N$-methyl pyrrolidine (NMP) in green, acetone in magenta, and cyrene (black circles) at $298 \mathrm{~K}$.

Table 4 Closest solvents to cyrene ranked in order of decreasing solvent similarity index (SSI) calculated at $298 \mathrm{~K}$

\begin{tabular}{ll}
\hline Solvent & SSI \\
\hline 2,4-Pentanedione & 0.018 \\
Methyl formate & 0.020 \\
Ethyl formate & 0.023 \\
4-Methoxybenzaldehyde & 0.025 \\
Pyrimidine & 0.027 \\
1-Methyl-2-pyrrolidinethione & 0.027 \\
Dimethylcyanamide & 0.029 \\
Ethyl acetoacetate & 0.029 \\
Dimethyl carbonate & 0.031 \\
Cinnamaldehyde & 0.031 \\
Methyl acetate & 0.032 \\
Acetophenone & 0.033 \\
Dimethylphthalate & 0.033 \\
2-Bromopyridine & 0.034 \\
Acetone & 0.036 \\
Diethyl malonate & 0.036 \\
Benzyl methyl ketone & 0.037 \\
4-Methyl-2-pentanone & 0.040 \\
Diethyl sulfite & 0.040 \\
Propionaldehyde & 0.040 \\
Diethyl carbonate & 0.040 \\
Ethyl acetate & 0.040 \\
Ethyl phenyl ketone & 0.041 \\
2,3-Butanedione & 0.043 \\
3-Bromopyridine & 0.043 \\
\end{tabular}

\section{Conclusion}

A key property in the selection of a solvent for a chemical transformation or formulation is the strength of the interactions with solute molecules, which in turn determines how the solutes will behave. The Surface Site Interaction Model for Liquids at Equilibrium (SSIMPLE) provides a method for calculating the free energy of solvation of a specific interaction site on the surface of a solute. The solvent is described as a set of Surface Site Interaction Points (SSIP), which describe the noncovalent interactions that can be made at any point on the molecular surface, and the solute is described as one SSIP, which would correspond to a single hydrogen bond donor or acceptor site on the surface of a polar solute. This representation can be used to calculate the free energy of solvation for all possible solute polarities, generating a unique solvation profile for any solvent or solvent mixture. These solvation profiles provide a quantitative tool for comparison of the solvation properties of two solvents, which we define as the Solvation Similarity Index. Values of SSI were calculated for all pairwise comparisons of 261 pure solvents at $298 \mathrm{~K}$, and the results were used to classify solvents into groups according to their solvation properties. Application of the SSI for understanding the solvation properties of binary solvent mixtures and for identification of alternative solvents are demonstrated. The SSI represents a new quantitative parameter that can be applied to complex solvent environments of arbitrary complexity and should have applications in assessing the expected properties of candidates for new solvents from sustainable sources.

\section{Conflicts of interest}

There are no conflicts to declare.

\section{Acknowledgements}

We acknowledge financial support from the Engineering and Physical Sciences Research Council (EPSRC) for an EPSRC doctoral training studentship (grant code EP/M506485/1) for M. Driver.

\section{Notes and references}

1 D. Prat, J. Hayler and A. Wells, Green Chem., 2014, 16, 4546-4551.

2 F. P. Byrne, S. Jin, G. Paggiola, T. H. M. Petchey, J. H. Clark, T. J. Farmer, A. J. Hunt, C. Robert McElroy and J. Sherwood, Sustainable Chem. Processes, 2016, 4, 7.

3 T. Lindvig, M. L. Michelsen and G. M. Kontogeorgis, Fluid Phase Equilib., 2002, 203, 247-260.

4 C. M. Hansen, Hansen Solubility Parameters: A User's Handbook, CRC Press, 2nd edn, 2007.

5 J. Lu, F. Yan and J. Texter, Prog. Polym. Sci., 2009, 34, 431-448.

6 Y. Kim, C. J. Cramer and D. G. Truhlar, J. Phys. Chem. A, 2009, 113, 9109-9114.

7 W. R. Fawcett and J. S. Jaworski, J. Phys. Chem., 1983, 87, 2972-2976.

8 E. D. Hughes and C. K. Ingold, J. Chem. Soc., 1935, 244.

9 M. H. Abraham, Pure Appl. Chem., 1985, 57, 1055-1064.

10 B. S. Akpa, C. D’Agostino, L. F. Gladden, K. Hindle, H. Manyar, J. McGregor, R. Li, M. Neurock, N. Sinha, E. H. Stitt, D. Weber, J. A. Zeitler and D. W. Rooney, J. Catal., 2012, 289, 30-41. 
11 R. K. Henderson, C. Jiménez-González, D. J. C. Constable, S. R. Alston, G. G. A. Inglis, G. Fisher, J. Sherwood, S. P. Binks and A. D. Curzons, Green Chem., 2011, 13, 854.

12 K. Alfonsi, J. Colberg, P. J. Dunn, T. Fevig, S. Jennings, T. A. Johnson, H. P. Kleine, C. Knight, M. A. Nagy, D. A. Perry and M. Stefaniak, Green Chem., 2008, 10, 31-36.

13 C. Jimenez-Gonzalez, C. S. Ponder, Q. B. Broxterman and J. B. Manley, Org. Process Res. Dev., 2011, 15, 912-917.

14 M. Tobiszewski, J. Namieśnik and F. Pena-Pereira, Green Chem., 2017, 19, 1034-1042.

15 J. H. Hildebrand and R. L. Scott, Solubility of non-electrolytes, Reinhold Pub, 1936.

16 R. Mannhold, G. I. Poda, C. Ostermann and I. V. Tetko, Calculation of molecular lipophilicity: State-of-the-art and comparison of $\log P$ methods on more than 96000 compounds, 2009.

17 C. Nieto-Draghi, G. Fayet, B. Creton, X. Rozanska, P. Rotureau, J.-C. de Hemptinne, P. Ungerer, B. Rousseau and C. Adamo, Chem. Rev., 2015, 115, 13093-13164.

18 R. E. Skyner, J. L. McDonagh, C. R. Groom, T. van Mourik and J. B. O. Mitchell, Phys. Chem. Chem. Phys., 2015, 17, 6174-6191.

19 G. Klopman, J.-Y. Li, S. Wang and M. Dimayuga, J. Chem. Inf. Model., 1994, 34, 752-781.

20 W. M. Meylan and P. H. Howard, J. Pharm. Sci., 1995, 84, 83-92.

21 W. M. Meylan and P. H. Howard, J. Pharm. Sci., 1995, 84, 83-92.

22 A. Leo, P. Y. C. Jow, C. Silipo and C. Hansch, J. Med. Chem., 1975, 18, 865-868.

23 P. Japertas, R. Didziapetris and A. Petrauskas, Quant. Struct.-Act. Relat., 2002, 21, 23-37.

24 P. Myrdal, G. H. Ward, R.-M. Dannenfelser, D. Mishra and S. H. Yalkowsky, Chemosphere, 1992, 24, 1047-1061.

25 S. Pinsuwan, P. B. Myrdal, Y. C. Lee and S. H. Yalkowsky, Chemosphere, 1997, 35, 2503-2513.

26 A. K. Ghose and G. M. Crippen, J. Comput. Chem., 1986, 7, 565-577.

27 M. Waldherr-Teschner, T. Goetze, W. Heiden, M. Knoblauch, H. Vollhardt and J. Brickmann, Advances in Scientific Visualization, Springer Berlin Heidelberg, Berlin, Heidelberg, 1992, pp. 58-67.

28 S. Wildman and G. Crippen, J. Chem. Inf. Model., 1999, 39, 868-873.

29 R. Wang, Y. Fu and L. Lai, J. Chem. Inf. Model., 1997, 2338, 615-621.

30 M. Junghans and E. Pretsch, Fresenius' J. Anal. Chem., 1997, 359, 88-92.

31 L. H. Hall and L. B. Kier, J. Chem. Inf. Model., 1995, 35, 1039-1045.

32 M. H. Abraham, J. Phys. Org. Chem., 1993, 6, 660-684.

33 M. H. Abraham, H. S. Chadha, J. P. Dixon and A. J. Leo, J. Phys. Org. Chem., 1994, 7, 712-716.

34 I. V. Tetko, V. Y. Tanchuk and A. E. P. Villa, J. Chem. Inf. Model., 2001, 41, 1407-1421.

35 R. Liu and D. Zhou, J. Chem. Inf. Model., 2008, 48, 542-549.
36 H.-F. Chen, Chem. Biol. Drug Des., 2009, 74, 142-147.

37 I. V. Tetko, I. Sushko, A. K. Pandey, H. Zhu, A. Tropsha, E. Papa, T. Öberg, R. Todeschini, D. Fourches and A. Varnek, J. Chem. Inf. Model., 2008, 48, 1733-1746.

38 J. Tomasi, B. Mennucci and R. Cammi, Chem. Rev., 2005, 105, 2999-3094.

39 W. C. Still, A. Tempczyk, R. C. Hawley and T. Hendrickson, J. Am. Chem. Soc., 1990, 112, 6127-6129.

40 W. D. Cornell, P. Cieplak, C. I. Bayly, I. R. Gould, K. M. Merz, D. M. Ferguson, D. C. Spellmeyer, T. Fox, J. W. Caldwell and P. A. Kollman, J. Am. Chem. Soc., 1995, 117, 5179-5197.

41 A. V. Marenich, R. M. Olson, C. P. Kelly, C. J. Cramer and D. G. Truhlar, J. Chem. Theory Comput., 2007, 3, 2011-2033.

42 C. J. Cramer and D. G. Truhlar, Acc. Chem. Res., 2008, 41, 760-768.

43 A. Klamt and G. Schüürmann, J. Chem. Soc., Perkin Trans. 2, 1993, 799-805.

44 A. Klamt, J. Phys. Chem., 1995, 2224-2235.

45 S.-T. Lin and S. I. Sandler, Ind. Eng. Chem. Res., 2002, 899-913.

46 S.-T. Lin and S. I. Sandler, Ind. Eng. Chem. Res., 2004, 43, 1322.

47 N. M. Garrido, I. G. Economou, A. J. Queimada, M. Jorge and E. A. Macedo, AIChE J., 2012, 58, 1929-1938.

48 N. M. Garrido, A. J. Queimada, M. Jorge, E. A. Macedo and I. G. Economou, J. Chem. Theory Comput., 2009, 5, 2436-2446.

49 D. L. Mobley and J. P. Guthrie, J. Comput.-Aided Mol. Des., 2014, 28, 711-720.

50 O. Beckstein, A. Fourrier and B. I. Iorga, J. Comput.-Aided Mol. Des., 2014, 28, 265-276.

51 G. Duarte Ramos Matos, G. Calabrò and D. L. Mobley, J. Chem. Theory Comput., 2019, 15, 3066-3074.

52 P. A. Kollman, I. Massova, C. Reyes, B. Kuhn, S. Huo, L. Chong, M. Lee, T. Lee, Y. Duan, W. Wang, O. Donini, P. Cieplak, J. Srinivasan, D. A. Case and T. E. Cheatham, Acc. Chem. Res., 2000, 33, 889-897.

53 B. R. Miller, T. D. McGee, J. M. Swails, N. Homeyer, H. Gohlke and A. E. Roitberg, J. Chem. Theory Comput., 2012, 8, 3314-3321.

54 S. Genheden and U. Ryde, J. Comput. Chem., 2010, 31, 837-846.

55 C. A. Hunter, Chem. Sci., 2013, 4, 1687-1700.

56 P. Anastas and J. Warner, Green Chemistry: Theory and Practice, Oxford University Press, 2000.

57 D. Prat, O. Pardigon, H. W. Flemming, S. Letestu, V. Ducandas, P. Isnard, E. Guntrum, T. Senac, S. Ruisseau, P. Cruciani and P. Hosek, Org. Process Res. Dev., 2013, 17, 1517-1525.

58 C. A. Hunter, Angew. Chem., Int. Ed., 2004, 43, 5310-5324.

59 D. Gurka and R. W. Taft, J. Am. Chem. Soc., 1969, 91, 4794-4801.

60 R. W. Taft, D. Gurka, L. Joris, P. R. Schleyer and J. W. Rakshys, J. Am. Chem. Soc., 1969, 91, 4801-4808.

61 M. H. Abraham and J. A. Platts, J. Org. Chem., 2001, 66, 3484-3491. 
62 P. Politzer, J. S. Murray and Z. Peralta-Inga, Int. J. Quantum Chem., 2001, 85, 676-684.

63 J. S. Murray and P. Politzer, Wiley Interdiscip. Rev.: Comput. Mol. Sci., 2011, 1, 153-163.

64 J. S. Murray and P. Politzer, Wiley Interdiscip. Rev.: Comput. Mol. Sci., 2017, 7, 1-10.

65 C. S. Calero, J. Farwer, E. J. Gardiner, C. A. Hunter, M. Mackey, S. Scuderi, S. Thompson and J. G. Vinter, Phys. Chem. Chem. Phys., 2013, 15, 18262-18273.

66 C. A. Hunter, Chem. Sci., 2013, 4, 834-848.

67 M. D. Driver, M. J. Williamson, J. Cook and C. A. Hunter, Chem. Sci., 2020, 11, 4456-4466.
68 J. Sherwood, M. De bruyn, A. Constantinou, L. Moity, C. R. McElroy, T. J. Farmer, T. Duncan, W. Raverty, A. J. Hunt and J. H. Clark, Chem. Commun., 2014, 50, 9650-9652.

69 F. Cao, T. J. Schwartz, D. J. Mcclelland, S. H. Krishna, J. A. Dumesic and G. W. Huber, Energy Environ. Sci., 2015, 8, 1808-1815.

70 K. L. Wilson, A. R. Kennedy, J. Murray, B. Greatrex, C. Jamieson and A. J. B. Watson, Beilstein J. Org. Chem., 2016, 12, 2005-2011.

71 L. Mistry, K. Mapesa, T. W. Bousfield and J. E. Camp, Green Chem., 2017, 19, 2123-2128. 